\title{
On the application of the image-guided ray tracing
}

Eduardo Filpo Ferreira da Silva, PETROBRAS

Copyright 2019, SBGf - Sociedade Brasileira de Geofísica

This paper was prepared for presentation during the $16^{\text {th }}$ International Congress of the Brazilian Geophysical Society held in Rio de Janeiro, Brazil, 19-22 August 2019.

Contents of this paper were reviewed by the Technical Committee of the $16^{\text {th }}$ International Congress of the Brazilian Geophysical Society and do not necessarily represent any position of the SBGf, its officers or members. Electronic reproduction or storage of any part of this paper for commercial purposes without the written consent of the Brazilian Geophysical Society is prohibited.

\section{Abstract}

I present some applications of a new ray-tracing system derived from an eikonal equation used in the development of some image-guided algorithms. The initial motivation was to accelerate the construction of traveltime tables for image-guided interpolation without any worry about the physical meaning of these rays. Indeed, I just use the ray theory to find some curves in order to solve a practical problem. The observation of the first obtained curves, immediately show me a wide range of applicability of this image-guided ray tracing system. The method is suitable for almost all applications that involve any kind of tracking of seismic events. The application examples presented are image guided interpolation, well log correlation, residual moveout picking for tomography, model building and image flattening.

\section{Introduction}

The term image-guided interpolation was introduced by Hale (2009) to refer a method specially designed to interpolate scattered data. The image-guided interpolation makes use of non-Euclidean distances in metric tensor fields that represent the coherence, orientations and shapes of features in image. The first step of this interpolation consists of computing time maps for each given scattering point, and selecting the minimum time for each image grid position.

Time maps are computed by solving the equation

$$
\nabla t(x) \cdot D(x) \nabla t(x)=1,
$$

which corresponds to an anisotropic eikonal equation. In this equation, $x$ is position vector, $t(x)$ represents the time map, and $D(x)$ is tensor field.

The tensor field $D(x)$ is equivalent to the diffusion tensor with continuity factor proposed by Fehmers and Höcker (2003) to filter images preserving faults and following the structure orientation. Weickert is the first author to use anisotropic diffusion for image processing in his dissertation, see Weickert, 1998.

The idea of deriving a ray-tracing system from the anisotropic eikonal equation associated with the diffusion tensor naturally appears when I think in an alternative method for travel time computation. Cerveny et al (2012) shows how to compute paraxial travel times from dynamic ray-tracing quantities for several source-receiver configurations in anisotropic inhomogeneous media. Waheed et al (2013) demonstrates that the paraxial approximation for travel time calculation could be very precise. My experience in working with traveltime tables for Kirchhoff migration shows that paraxial approximation is not only more precise than fast marching method, but also is much more efficient.

A classical approach to derive the kinematic and the dynamic ray-tracing system for any kind of media is to start representing the eikonal equation in its Hamiltonian form, and then derive the equations as described in Cerveny (2001).

\section{Method}

I start representing the "diffusion" eikonal equation in its Hamiltonian form

$$
H(x, p)=\frac{1}{2}[p \cdot D(x) p-1] .
$$

Where $p=\nabla t(x)$ is equivalent to the slowness vector in the conventional ray-tracing. According to Cerveny (2001), there are two systems:

$$
\left\{\begin{array}{l}
\frac{d x}{d u}=\nabla_{p} H \\
\frac{d p}{d u}=-\nabla_{x} H \\
\frac{d t}{d u}=p^{T} \nabla_{p} H
\end{array}\right.
$$

and,

$$
\frac{d \Pi}{d u}=\left[\begin{array}{cc}
\nabla_{p} \nabla_{x}^{T} H & \nabla_{p} \nabla_{x}^{T} H \\
-\nabla_{x} \nabla_{x}^{T} H & -\nabla_{x} \nabla_{x}^{T} H
\end{array}\right] \Pi
$$

Equation (3) is the kinematic ray-tracing system composed of five differential equations for the 2D case, or seven for 3D, being $u$ some parameter along the ray. For a specific $u$, the solution of this system is the position $x$, the direction $p$ and the traveltime $t$.

Equation (4) is the dynamic ray-tracing system and its solution is the propagator matrix $\Pi$, which contains the quantities necessary to obtain the paraxial traveltime at the vicinity of the ray.

In 2D case, the diffusion tensor is a positive-definite symmetric matrix: 


$$
D=\left[\begin{array}{ll}
d_{11} & d_{12} \\
d_{12} & d_{22}
\end{array}\right],
$$

And the Hamiltonian is

$$
H=\frac{1}{2}\left(d_{11} p_{1}^{2}+2 d_{12} p_{1} p_{2}+d_{22} p_{2}^{2}-1\right),
$$

Being $p_{1}$ and $p_{2}$ the components of the vector $p$, and $d_{11}, d_{12}$ and $d_{22}$ are the components of the diffusion tensor.

\section{Examples}

The first application is the construction of time maps for image guided interpolation, IGI. The algorithm proposed by Hale (2009) to interpolate sparse measures consists of two steps: i) calculation of minimum time map, and ii) structure oriented smoothing of the neighbor interpolation using a metric tensor obtained from the minimum time map.

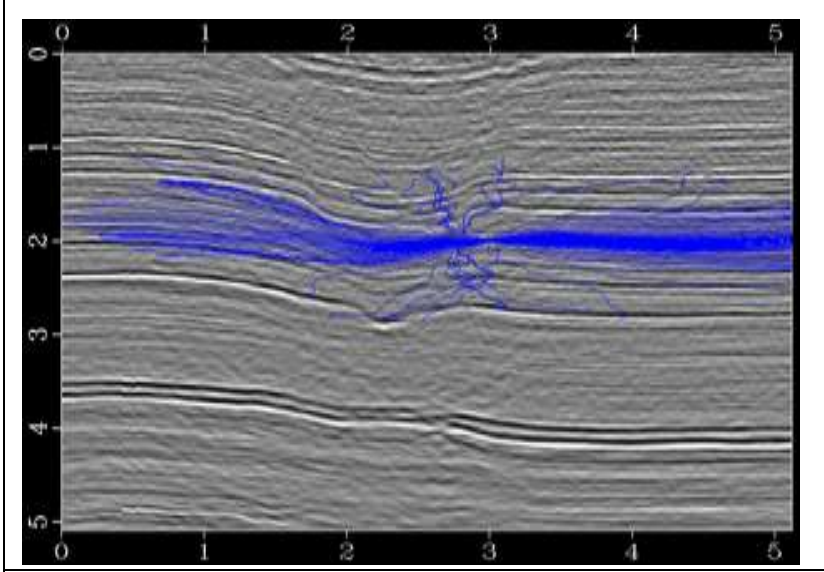

Figure 1a) - Bundle of rays, in blue, with uniform angular distribution superimposed to the image used to create the diffusion tensor.

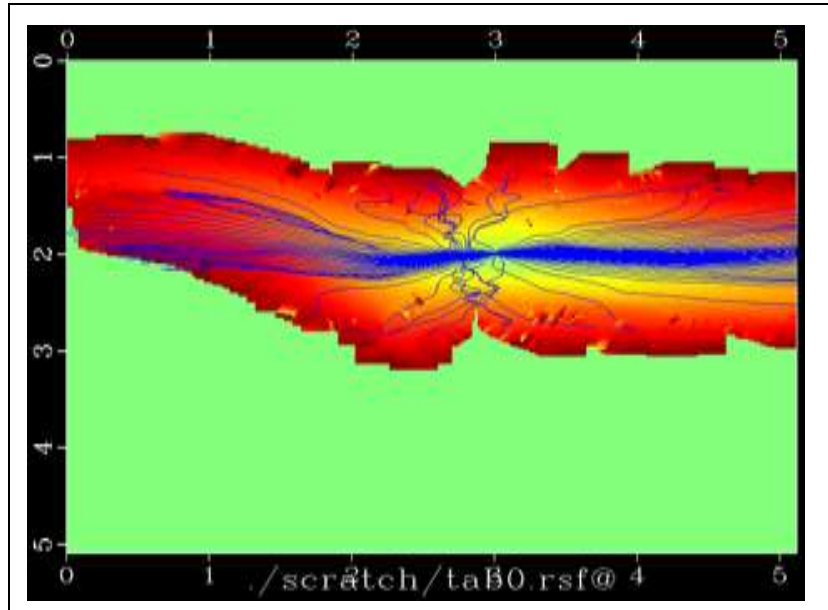

Figure 1b) - Bundle of rays, in blue, superimposed to traveltime map obtained by paraxial approximation.
Figure 1a) shows a bundle of rays superimposed to the seismic image used as a guide for interpolation. These rays are traced with uniform distribution of takeoff angles and with the same number of steps, pay attention how they go further in the structure direction and how they tend to be aligned to the seismic reflectors. Figure 1b) shows the same bundle of rays superimposed to the travel time map obtained by paraxial approximation. Remember that paraxial approximation computation requires the knowledge of certain quantities obtained with dynamic ray-tracing. Figure 2 shows the minimum time map for a set of sparse points, in blue, with measures to be interpolated.

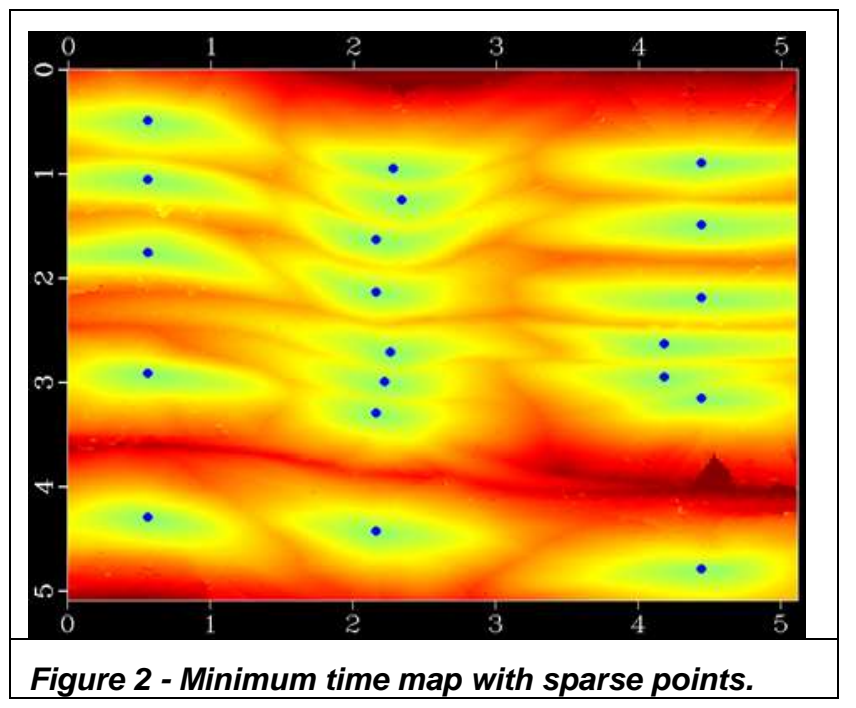

The other applications examples are based in a 2D image-guided wavefront construction algorithm, referred as IGWFC2D along this abstract. In general, wavefront construction algorithms try to keep the same density of rays in the space, so they are equipped with functionalities for creation and destruction of rays. The IGWFC2D algorithm involves only kinematic ray-tracing and it has only a ray destroyer function. This function avoids that rays cross each other. Every time two rays are closer than a threshold distance, this function freeze one of them, keeping that one whose direction is closer to the eigen-vector with greater eigen-value.

Among the applications of the IGWFC2D algorithm are: automatic residual moveout picking in image gathers for tomography, automatic tracking of seismic horizons, welllog interpolation, velocity function interpolation, model building and flattening of seismic images. Also, it could be used as auxiliary tool in geological features identification in seismic interpretation.

Figure 3 illustrates the use of IGWFC2D for well log interpolation. The well logs are located at each extreme of the image 3a), the interpolated value is a weighted average of the two logs, being the weight the inverse of measured time along rays coming from each side. Observe in $3 b$ ) how the interpolated values naturally follow the seismic horizons. The same strategy can be used to interpolate seismic velocities or any other property. 


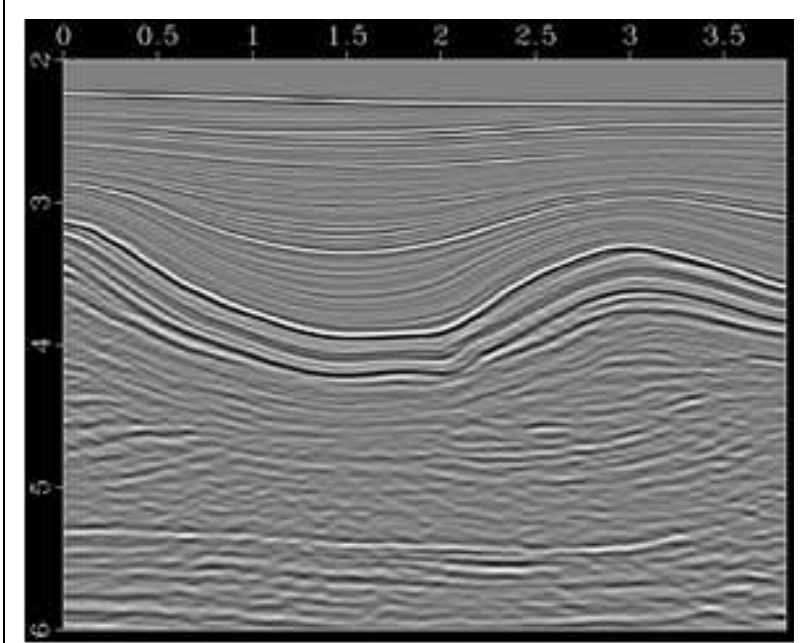

Figure 3a) - Seismic sections used as a guide for the well log interpolation example.

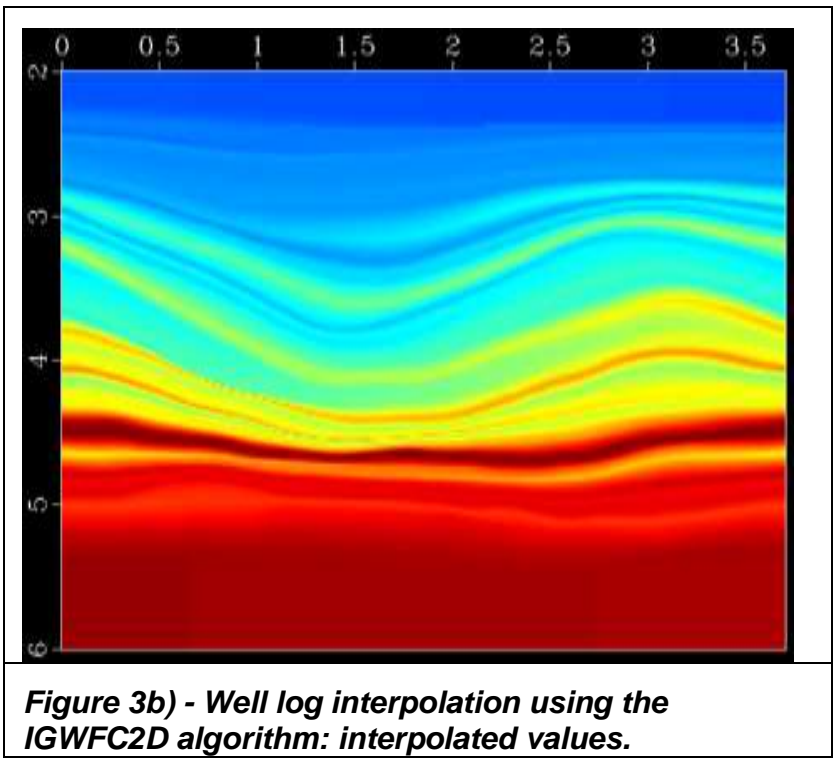

Figure 4a) and 4b) illustrate how the IGWFC2D algorithm could be used to create very detailed seismic models in a very efficient way. The first shows the rays superimposed to the image used to obtain the diffusion tensor. Observe that the set of rays follow a very complex pattern that is highly correlated with the geological features observed in the image. The second is a model created just by filling the space with the ray index. A low-frequency model for inversion could be easily created just by filling the space with any property related to velocity instead of a simple index.

Figures $5 \mathrm{a}$ to $5 \mathrm{c}$ illustrate how the IGWFC2D algorithm could be used to perform flattening of seismic images: a) is a set of selected rays for vertical shift calculation, b) is the calculated vertical shift map, and $c$ ) is the flattened image.

Figures $6 a$ and $6 b$ are examples of the use of the IGWFC2D algorithm to evaluate residual moveout for tomography. The first is a synthetic gather; observe that rays always follow the seismic event, even when they started in a very low amplitude region. It happens because diffusion tensors are generated from amplitude derivatives, not amplitude itself. The second is a common image gather from field dataset. Observe that the algorithm is capable to notice truncated events and even in this case provides a good picking solution.

\section{Conclusions}

I present a new method for trace curves with image control. I refer to these curves as image-guided rays, because we use a system of partial differential equations obtained from the ray theory. In this context, the diffusion tensor plays the role of an anisotropic velocity field. Although I had used only seismic images in our examples, the method could be applied to any image, since the diffusion tensor had been properly generated. There is no restriction for applying the methodology in tridimensional images.

I present several successful application examples of image guided ray-tracing, among of them are image guided interpolation, well log correlation, picking of residual moveout for tomography, model building and image flattening.

\section{Acknowledgments}

I thank PETROBRAS for supporting this research and for permitting this publication. Also, I thank my colleagues Marcos Machado and Luiz Alberto for fruitful discussion and valuable suggestions.

\section{References}

CERVENY, V., 2001, Seismic Ray Theory, Cambridge: Cambridge Ubiv. Press.

CERVENY, V., IVERSEN E., Psencik, I., 2012, Two-point paraxial traveltimes in an inhomogeneous anisotropic medium: Geophysical Journal International, 189, 1597 1610.

FEHMERS, G.C., HÖCKER, C.F.W., 2003, Fast structural interpretations with structure-oriented filtering: Geophysics, 68, 1286-1293.

HALE, D., 2009, Image-guided blended interpolation: CWP Report, 258-260.

WAHEED, U., PSENSIK, I., CERVENY, V., IVERSEN, E., ALKHALIFAH, T.,2013, Two-point paraxial traveltime formula for inhomogeneous isotropic and anisotropic media: Tests of accuracy: Geophysics, 78, 65-80.

WEICKERT,J., 1998, Anisotropic diffusion in image processing: Teubner Verlag. 


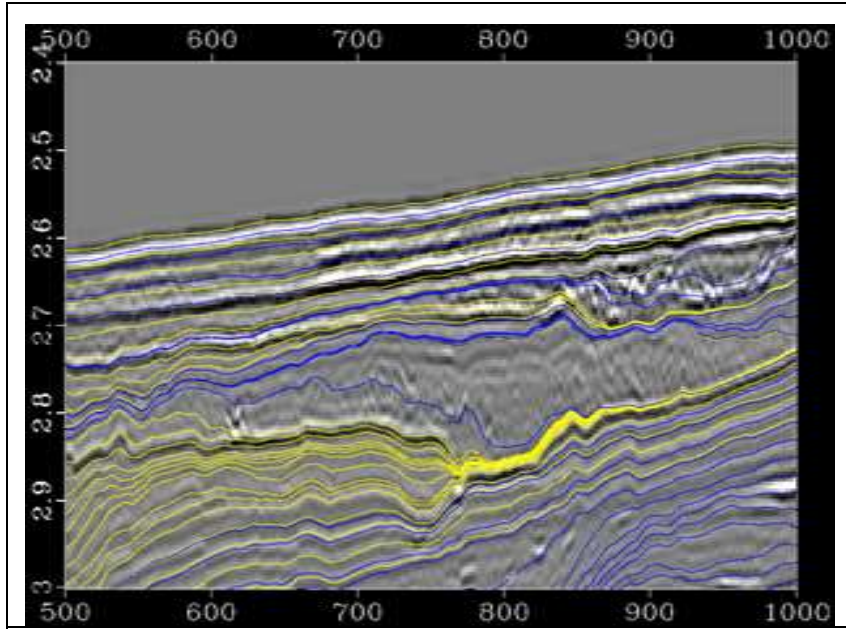

Figure 4a) - Example of use of IGWFC2D algorithm to create seismic models: set of rays superimposed to the image, rays coming from the left in yellow, and coming from the right in blue

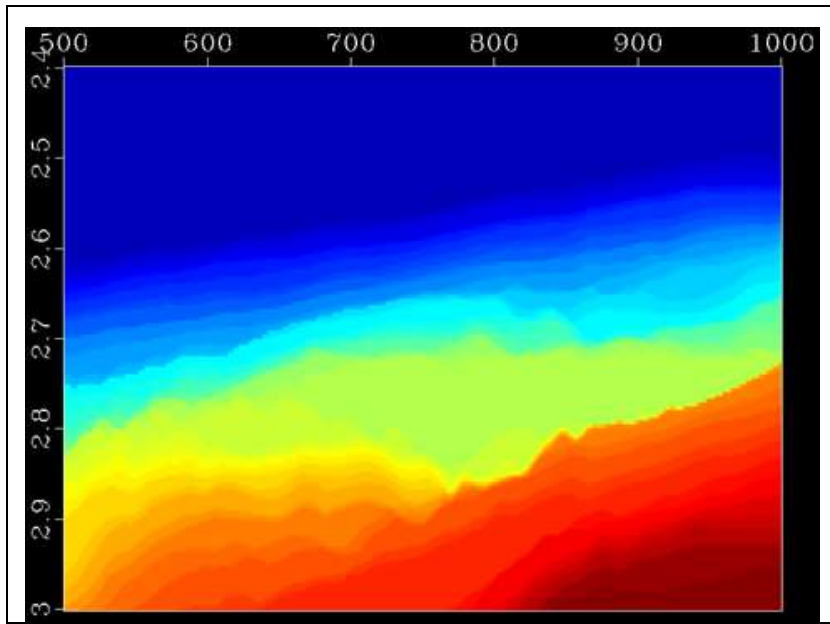

Figure 4b) - Example of use of IGWFC2D algorithm to create seismic models: seismic model created by filling space with rays' index.

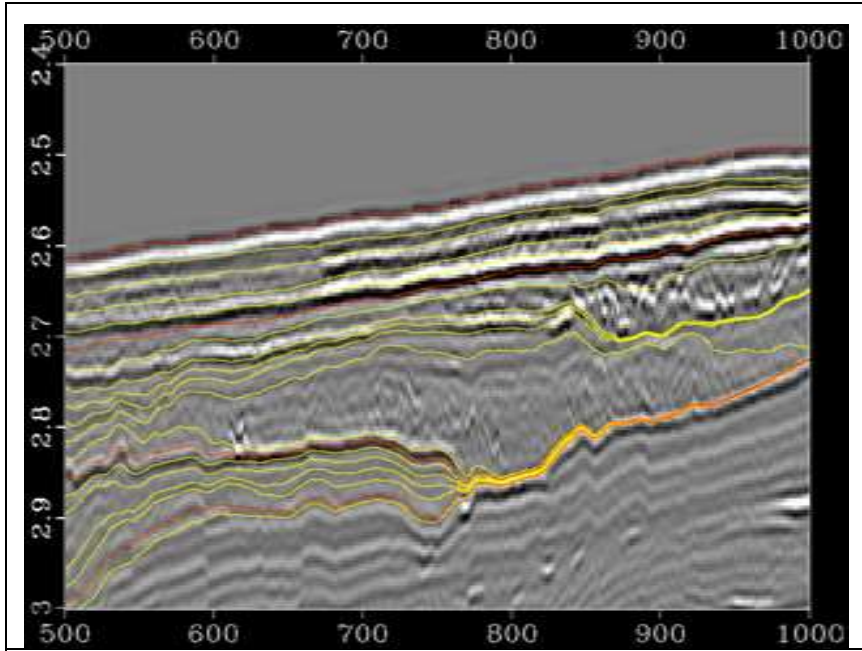

Figure 5a) - Example of use of IGWFC2D algorithm for automatic flattening of seismic images: set of rays used to create a vertical shift map.

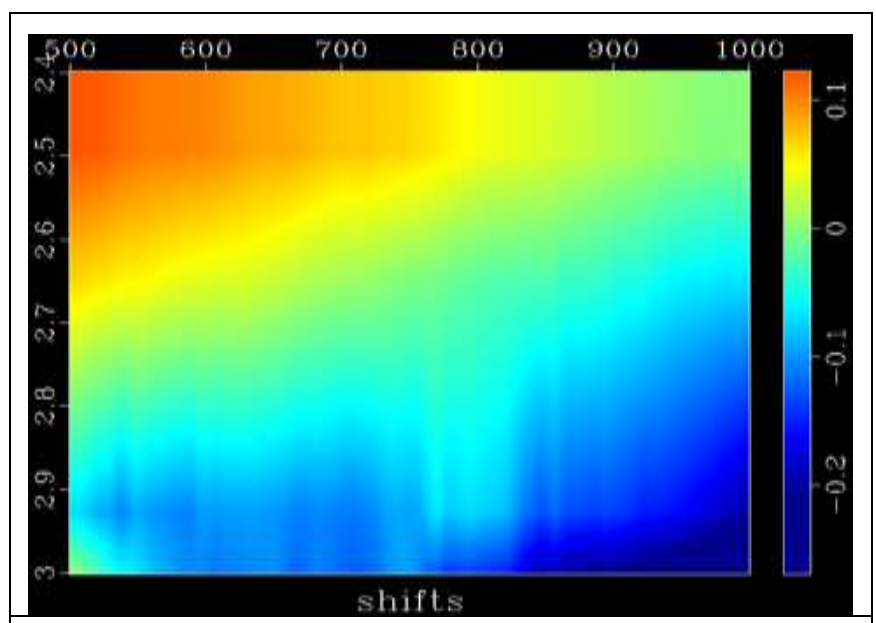

Figure 5b) - Example of use of IGWFC2D algorithm for automatic flattening of seismic images: vertical shift map. 


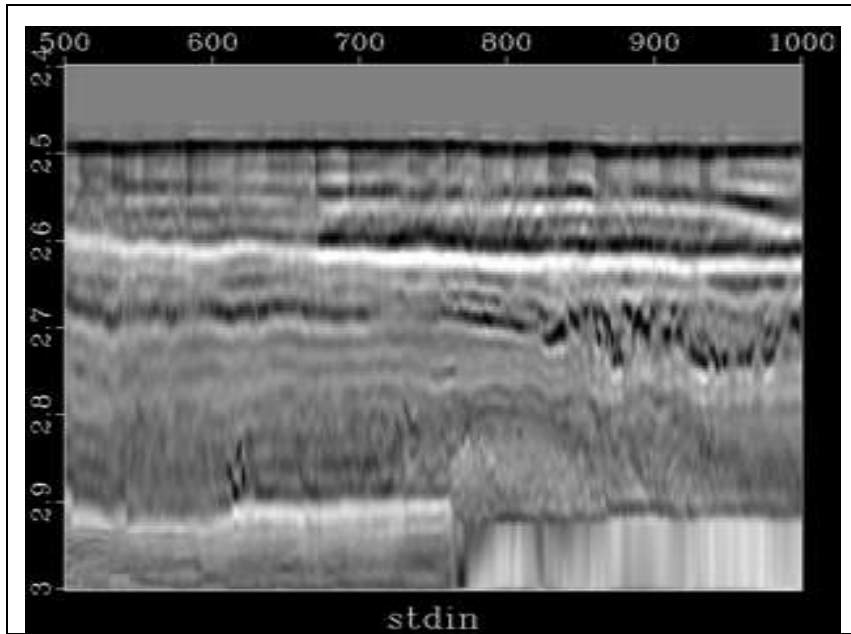

Figure 5c) - Example of use of IGWFC2D algorithm for automatic flattening of seismic images: c) Flattened image.

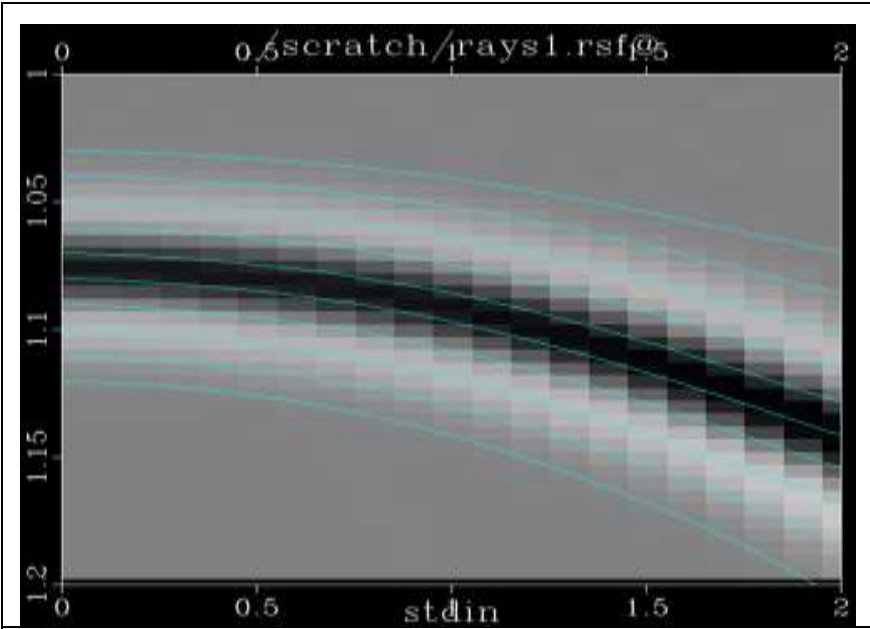

Figure 6a) - Example of use of IGWFC2D algorithm for automatic picking of residual moveout: synthetic dataset.

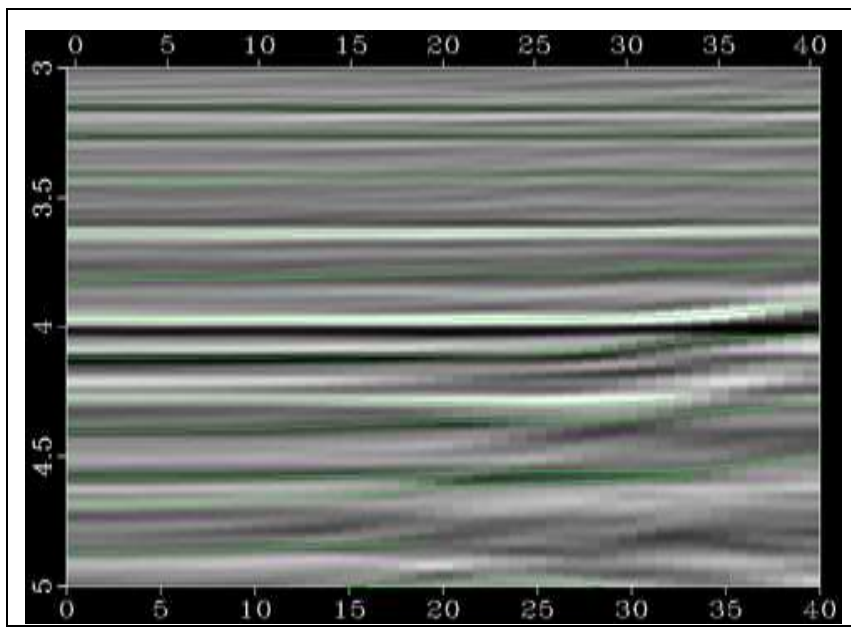

Figure 6b) - Example of use of IGWFC2D algorithm for automatic picking of residual moveout: field image gather. 
